\title{
COINVARIANTS OF NILPOTENT SUBALGEBRAS OF THE VIRASORO ALGEBRA AND PARTITION IDENTITIES
}

\author{
BORIS FEIGIN AND EDWARD FRENKEL \\ Dedicated to I.M. Gelfand on his 80th birthday
}

\section{InTRODUCTION}

Let $V_{p, q}^{m, n}$ be the irreducible representation of the Virasoro algebra $\mathcal{L}$ with central charge $c_{p, q}=1-6(p-q)^{2} / p q$ and highest weight $h_{m, n}=$ $\left[(n p-m q)^{2}-(p-q)^{2}\right] / 4 p q$, where $p, q>1$ are relatively prime integers, and $m, n$ are integers, such that $0<m<p, 0<n<q$. For fixed $p$ and $q$ the representations $V_{p, q}^{m, n}$ form the $(p, q)$ minimal model of the Virasoro algebra [1].

For $N>0$ let $\mathcal{L}_{N}$ be the Lie subalgebra of the Virasoro algebra, generated by $L_{i}, i<-N$. There is a map from the Virasoro algebra to the Lie algebra of polynomial vector fields on $\mathbb{C}^{*}$, which takes $L_{i}$ to $z^{-i+1} \frac{\partial}{\partial z}$, where $z$ is a coordinate. This map identifies $\mathcal{L}_{N}$ with the Lie algebra of vector fields on $\mathbb{C}$, which vanish at the origin along with the first $N+1$ derivatives. The Lie algebra $\mathcal{L}_{2 N}$ has a family of deformations $\mathcal{L}\left(p_{1}, \ldots, p_{N+1}\right)$, which consist of vector fields, vanishing at the points $p_{i} \in \mathbb{C}$ along with the first derivative.

For a Lie algebra $g$ and a $g$-module $M$ we denote by $H(g, M)$ the space of coinvariants (or 0th homology) of $g$ in $M$, which is the quotient $M / g \cdot M$, where $g \cdot M$ is the subspace of $M$, linearly spanned by vectors $a \cdot x, a \in g, x \in M$. We will prove the following result.

Theorem 1. For any irreducible representation $V$ of the $(2,2 r+1)$ minimal model

$$
\operatorname{dim} H\left(\mathcal{L}_{2 N}, V\right)=\operatorname{dim} H\left(\mathcal{L}\left(p_{1}, \ldots, p_{N+1}\right), V\right) .
$$

This statement was proved in [2] for $N=2$ and irreducible representations of general minimal models.

As an application, we will give a new proof of the Gordon identities, which relies on two different computations of the characters of the irreducible representations of the $(2,2 r+1)$ minimal models.

Research of the second author was supported by a Junior Fellowship from the Harvard Society of Fellows and by NSF grant DMS-9205303. 
Let us note that a priori $\operatorname{dim} H\left(\mathcal{L}_{2 N}, V\right) \geq \operatorname{dim} H\left(\mathcal{L}\left(p_{1}, \ldots, p_{N+1}\right), V\right)$. Indeed, if we have a family of Lie algebras, then the dimension of homology is the same for generic points of the space of parameters of the family, but it may increase at special points.

Each minimal model of the Virasoro algebra associates to a punctured complex curve, with a representation inserted at each puncture, a linear space, which is called the space of conformal blocks. This space can be defined as the space of coinvariants of the Lie algebra of meromorphic vector fields on this curve, which are allowed to have poles only at the punctures, in the tensor product of these representations (cf. [1], mathematical aspects of this correspondence are treated in detail in [2], [3]).

In this language, the space $H\left(\mathcal{L}\left(p_{1}, \ldots, p_{N+1}\right), V_{p, q}^{m, n}\right)$ is isomorphic to the direct sum of the spaces of conformal blocks, associated to the projective line with punctures $p_{1}, \ldots, p_{N+1}$ with all possible insertions from the $(p, q)$ minimal model, and $\infty$ with the insertion of $V_{p, q}^{m, n}$. The dimension of this space can be calculated by a Verlinde type argument by deforming our curve - the projective line with $N+2$ punctures - to a joint union of $N$ projective lines, each with 3 punctures. Under this deformation the dimension of the homology does not change [2], [3], and can therefore be reduced to the well-known result on the dimensions of the spaces of conformal blocks, associated to the projective line with 3 insertions - the fusion coefficients.

This gives an explicit formula for $\operatorname{dim} H\left(\mathcal{L}\left(p_{1}, \ldots, p_{N+1}\right), V_{p, q}^{m, n}\right)$, which represents a lower bound for $\operatorname{dim} H\left(\mathcal{L}_{2 N}, V_{p, q}^{m, n}\right)$. We can also obtain an upper bound by a different method.

This method is based on the calculation of the annihilating ideal of a minimal model [4]. Let us recall the definition of the annihilating ideal. For $c \in \mathbb{C}$ let $M_{c}$ be the vacuum Verma module with central charge $c$. It is generated by a vector $v_{c}$, such that $L_{i} v_{c}=0, i \geq-1$. This representation has the structure of a vertex operator algebra (VOA) [5], which acts on any irreducible module $M$ of the Virasoro algebra with the same central charge. It means that each vector of $M_{c}$ defines a local current (or field), which is a formal power series in $z$ and $z^{-1}$, whose Fourier coefficients are linear operators, acting on $M$. These local currents can be constructed as follows. Consider the projective line $\mathbb{C P}^{1}$ with three punctures: 0 and $\infty$ with the insertions of $M$, and $z$ with the insertion of $M_{c}$. The space of conformal blocks is onedimensional in this case. In other words, the space of coinvariants of the Lie algebra $\mathcal{A}(0, z)$ of meromorphic vector fields on $\mathbb{C P}^{1}$, which are allowed to have poles only at $0, \infty$ and $z$, in $M \otimes M \otimes M_{c}$, is one-dimensional. It has a canonical generator - the projection of the 
tensor product of the highest weight vectors. The dual to this generator defines for each vector of $M_{c}$ a linear operator from $M$ to $M$, depending on $z$, which is our local current.

These local currents can be constructed explicitly. The monomials

$$
\left\{L_{-m_{1}} \ldots L_{-m_{l}} v_{c} \mid m_{1} \geq m_{2} \geq \cdots \geq m_{l}>1\right\}
$$

linearly span $M_{c}$. The corresponding local currents are equal to

$$
\frac{1}{\left(m_{1}-2\right) !} \ldots \frac{1}{\left(m_{l}-2\right) !}: \partial_{z}^{m_{1}-2} T(z) \ldots \partial_{z}^{m_{l}-2} T(z):
$$

where $T(z)=\sum_{i \in \mathbb{Z}} L_{i} z^{-i-2}$. The Fourier components of thus defined local currents form a Lie algebra $U_{c}(\mathcal{L})_{l o c}$, which is called the local completion of the universal enveloping algebra of the Virasoro algebra with central charge $c$ [6], [4]. They act on any representation of $\mathcal{L}$, which has the same central charge.

The module $M_{c}$ is irreducible, if and only if $c$ is not equal to $c_{p, q}$. However, if $c=c_{p, q}$, then $M_{c}$ contains a (unique) singular vector. The quotient of $M_{c_{p, q}}$ by the submodule, generated by this singular vector, is isomorphic to $V_{p, q}^{1,1}$. This representation, which is called the vacuum representation of the $(p, q)$ minimal model, defines another VOA, which is the quotient of the VOA of $M_{c_{p, q}}$ in the appropriate sense [7]. An irreducible representation $M$ of the Virasoro algebra with central charge $c_{p, q}$ is a module over this VOA [7], if and only if the space of coinvariants of the Lie algebra $\mathcal{A}(0, z)$ in $M \otimes M \otimes V_{p, q}^{1,1}$ is one-dimensional. An explicit computation of the dimension of this space, which was made in [2], tells us that it is so, if and only if $M$ is an irreducible representation of the $(p, q)$ minimal model.

The Fourier components of the local current, corresponding to the singular vector of $M_{c_{p, q}}$, generate an ideal in $U_{c_{p, q}}(\mathcal{L})_{l o c}$, which is called the annihilating ideal of the $(p, q)$ minimal model. Any element of this ideal acts trivially on any representation of the $(p, q)$ minimal model.

Imposing this condition with respect to the generators of the annihilating ideal immediately leads to certain linear relations among the monomial elements of $V_{p, q}^{m, n}$. This allows to estimate the dimension of the coinvariants $H_{0}\left(\mathcal{L}_{2 N}, V_{p, q}^{m, n}\right)$ from above.

The peculiarity of the $(2,2 r+1)$ models is that this upper bound coincides with the lower bound, and is therefore exact. This proves Theorem 1 and leads to a nice combinatorial description of the minimal representations of these models. Namely, as a $\mathbb{Z}$-graded linear space, such a representation is isomorphic to the quotient of the space of polynomials in infinitely many variables by a certain monomial ideal. Thus we obtain an expression for the character of this module, which 
coincides with the right hand side of one of the Gordon identities. But it is known that this character is equal to the left hand side of the identity. Hence, we obtain a new proof of the Gordon identities.

Implementing this program for other minimal models would lead to a result, analogous to Theorem 1, as well as to nice character formulas for irreducible representations. However, for general minimal models, for which the representations are "smaller" and the structure of the annihilating ideal is more complicated, one should impose extra conditions, corresponding to other elements of the annihilating ideal, to obtain the exact upper bound.

\section{LOWER BOUND: GENUS 0 CONFORMAL BLOCKS}

In this section we will calculate the dimension of $H\left(\mathcal{L}\left(p_{1}, \ldots, p_{N+1}\right), V\right)$ for an irreducible representation $V$ of a $(p, q)$ minimal model.

Denote by $\mathcal{A}\left(p_{1}, \ldots, p_{N+1}\right)$ the Lie algebra of meromorphic vector fields on $\mathbb{C P}^{1}$, which are allowed to have poles only at the distinct points $p_{1}, \ldots, p_{N+1} \in \mathbb{C}$, and $\infty$. There is an embedding of this Lie algebra into the direct sum of $N+2$ Virasoro algebras, and thus it acts on tensor products $V_{1} \otimes \ldots \otimes V_{N+2}$ of $N+2$ reprersentations of the Virasoro algebra with the same central charge.

Let us fix central charge $c_{p, q}$ and an irreducible representation $V=$ $V_{N+2}$ of the $(p, q)$ minimal model. The following statement follows from the general results on computation of conformal blocks, outlined in Section 5 of [2] (cf. [3] for details).

Proposition 2. (1) $\operatorname{dim} H\left(\mathcal{L}\left(p_{1}, \ldots, p_{N+1}\right), V\right)=\sum \operatorname{dim} H\left(\mathcal{A}\left(p_{1}, \ldots, p_{N+1}\right), V_{1} \otimes\right.$ $\left.\ldots \otimes V_{N+1} \otimes V\right)$, where the sum is taken over all $(N+1)$-tuples $V_{1}, \ldots, V_{N+1}$ of irreducible representations of the $(p, q)$ minimal model.

(2) $\operatorname{dim} H\left(\mathcal{A}\left(p_{1}, \ldots, p_{N+1}\right), V_{1} \otimes \ldots \otimes V_{N+1} \otimes V\right)=\sum \operatorname{dim} H\left(\mathcal{A}\left(p_{1}, \ldots, p_{N}\right), V_{1} \otimes\right.$ $\left.\ldots \otimes V_{N} \otimes W\right) \cdot \operatorname{dim} H\left(\mathcal{A}(0,1), W \otimes V_{N+1} \otimes V\right)$, where the sum is taken over all irreducible representations $W$ of the $(p, q)$ minimal model.

Using this result, we can calculate $\operatorname{dim} H\left(\mathcal{L}\left(p_{1}, \ldots, p_{N+1}\right), V\right)$ by induction.

Let $V_{1}, \ldots, V_{s}$ be the set of all irreducible representations of the $(p, q)$ minimal model $(s=(p-1)(q-1) / 2)$, and let $\mathbf{u}_{N}$ be the $s$-vector, whose components are $u_{N}^{i}=\operatorname{dim} H\left(\mathcal{L}\left(p_{1}, \ldots, p_{N+1}\right), V_{i}\right), i=1, \ldots, s$. Put $c_{i j k}=\operatorname{dim} H\left(\mathcal{A}(0,1), V_{i} \otimes V_{j} \otimes V_{k}\right)$. The numbers $c_{i j k}$ are usually called the fusion coefficients. They define the fusion algebra of this minimal model, which has generators $g_{i}, 1 \leq i \leq s$ and relations $g_{i} \cdot g_{j}=$ $\sum_{k} c_{i j k} g_{k}$ 
Let us introduce the $s \times s$ matrix $\mathcal{M}=\mathcal{M}_{p, q}$, whose $(i, j)$ th entry is equal to $\sum_{k} c_{i j k}$. This matrix can be interpreted as the matrix of action of the sum $\sum_{k} g_{k}$ on the fusion algebra.

According to Proposition 2, $u_{N}^{i}=\sum_{j, k} c_{i j k} u_{N-1}^{j}$. This gives us the recursion relation for $\mathbf{u}_{N}$ :

$$
\mathbf{u}_{N}=\mathcal{M} \mathbf{u}_{N-1} .
$$

For any $i$, the representation $V_{i}$ is generated from the highest weight vector $v_{i}$ by the action of the Lie algebra $\mathcal{L}_{0}$, hence $u_{0}^{i}=1$. Therefore, we obtain the formula

$$
\mathbf{u}_{N}=\mathcal{M}^{N} \mathbf{u}_{0}
$$

where $\mathbf{u}_{0}^{t}=[1,1, \ldots, 1]$.

This formula enables us to calculate the dimensions $u_{N}^{i}$ explicitly by diagonalizing the matrix $\mathcal{M}$.

In the rest of the paper we will focus on the $(2,2 r+1)$ models. In such a model we have $r$ irreducible representations $V_{i}=V_{2,2 r+1}^{1, i}, i=1, \ldots, r$. The $(i, j)$ th entry of the corresponding $r \times r$ matrix $\mathcal{M}$ is equal to $\min \{i, j\}$. This matrix is equal to the square of the matrix

$$
M_{r}(x)=\left(\begin{array}{ccccc}
0 & 0 & \ldots & 0 & 1 \\
0 & 0 & \ldots & 1 & 1 \\
\ldots & \ldots & \ldots & \ldots & \ldots \\
0 & 1 & \ldots & 1 & 1 \\
1 & 1 & \ldots & 1 & 1
\end{array}\right) .
$$

This fact has the following interpretation: in the fusion algebra of this model $\sum_{k} g_{k}$ is equal to the square of $g_{r}$, and the action of $g_{r}$ is given by matrix $M_{r}$.

Let us introduce vectors $\mathbf{x}_{i}$, whose $i$ th component is equal to 1 , and all other component are equal to 0 . Since $\mathbf{u}_{0}=M_{r}^{2} \mathbf{x}_{1}$, we have $u_{N}^{i}=$ $\mathbf{x}_{i}^{t} M_{r}^{2 N+2} \mathbf{x}_{1}$. This gives us a lower bound for $v_{N}^{i}=\operatorname{dim} H\left(\mathcal{L}_{2 N}, V_{i}\right)$.

Proposition 3. $v_{N}^{i} \geq \mathbf{x}_{i}^{t} M_{r}^{2 N+2} \mathbf{x}_{1}$.

In the next Section we will show that this bound is exact.

\section{UPPER BOUND: THE ANNIHILATING IDEAL}

The annihilating ideal of the $(2,2 r+1)$ minimal model is the ideal of the Lie algebra $U_{c_{2,2 r+1}}(\mathcal{L})_{l o c}$, generated by the Fourier components of the local current, corresponding to the singular vector of the vacuum Verma module $M_{c_{2,2 r+1}}$. All elements of this ideal act by 0 on any irreducible representation of the $(2,2 r+1)$ minimal model. In particular, 
the generators of the ideal act by 0 . This leads to certain relations between vectors in the irreducible minimal representations, as explained in [四].

Introduce a filtration on the irreducible representation $V_{i}$ of the $(2,2 r+1)$ models as follows: $0 \subset V_{i}^{0} \subset V_{i}^{1} \subset \ldots \subset V_{i}^{\infty}=V_{i}$, where $V_{i}^{k}$ is linearly spanned by the monomials $L_{-m_{1}} \ldots L_{-m_{l}} v_{i}$, such that $m_{1} \geq m_{2} \geq \cdots \geq m_{l} \geq 1$, and $l \leq k$. Let $\Omega_{i}$ be the adjoint graded space:

$$
\Omega_{i}=\oplus_{k \geq 0} \Omega_{i}^{k}, \quad \Omega_{i}^{k}=V_{i}^{k} / V_{i}^{k-1} .
$$

The space $\Omega_{i}$ is a polynomial algebra in variables $a_{j}, j>0$, where $a_{j}$ is the image of $L_{-j} v_{i}$.

The symbol of the singular vector of $M_{c_{2,2 r+1}}$ is equal to $L_{-2}^{r} v_{0}$. According to formula (11), the symbol of the corresponding local current is equal to : $T(z)^{r}$ :. Hence the symbols of the Fourier components of this current are given by the formula

$$
\sum_{j_{1}+\ldots+j_{r}=n}: L_{-j_{1}} \ldots L_{-j_{r}}: .
$$

Their action on $\Omega_{i}$ is given by the multiplication by

$$
S_{n}=\sum_{j_{k}>0 ; j_{1}+\ldots+j_{r}=n} a_{j_{1}} \ldots a_{j_{r}}
$$

for $n \geq r$, and 0 for $n<r$.

It is known that $V_{i}$ is the quotient of the Verma module with highest weight $h_{1, i}$ by the maximal submodule, generated by two singular vectors. The symbol of one of them is equal to $L_{-1}^{i} v_{i}$. This gives us a surjective map from the quotient $\Omega_{i}^{\prime}$ of $\mathbb{C}\left[a_{j}\right]_{j>0}$ by the ideal $I_{i}$ generated by $S_{n}, n>r$, and $a_{1}^{i}$, to $\Omega_{i}$.

Let $\Omega_{i}^{\text {mon }}$ be the quotient of $\mathbb{C}\left[a_{j}\right]_{j>0}$ by the ideal $I_{i}^{\text {mon }}$, generated by the monomials $a_{j}^{u} a_{j+1}^{v}, 0 \leq u<r, u+v=r, j>0$, and $a_{1}^{i}$.

We can introduce a $\mathbb{Z}$-grading on the module $V_{i}$ by putting $\operatorname{deg} v_{i}=$ $0, \operatorname{deg} L_{-j}=j$. The space $\Omega_{i}$ inherits this grading. We can also introduce a compatible $\mathbb{Z}$-grading on the space $\mathbb{C}\left[a_{j}\right]_{j>0}$ by putting $\operatorname{deg} a_{-j}=j$. The spaces $\Omega_{i}^{\prime}$ and $\Omega_{i}^{\text {mon }}$ inherit this grading.

For any $\mathbb{Z}$-graded linear space $V=\oplus_{n \geq 0} V(n)$, such that $\operatorname{dim} V(n) \leq$ $\infty$, let ch $V=\sum_{n \geq 0} \operatorname{dim} V(n) q^{n}$ be its character. We will write ch $V \leq$ $\operatorname{ch} V^{\prime}$, if $\operatorname{dim} V(n) \leq \operatorname{dim} V^{\prime}(n)$ for any $n \geq 0$.

We have

$$
\operatorname{ch} V_{i}=\operatorname{ch} \Omega_{i} \leq \operatorname{ch} \Omega_{i}^{\prime} \leq \operatorname{ch} \Omega_{i}^{\text {mon }} .
$$

The last inequality follows from the fact that each of the generators $S_{n}$ of the ideal $I_{i}$ has as a summand one and only one generator of the ideal $I_{i}^{\text {mon }}$, namely, $a_{j}^{u} a_{j+1}^{v}$ is a summand of $S_{r j+v}$. 
Now, by the construction of our filtration, the character of coinvariants $H\left(\mathcal{L}_{2 N}, V_{i}\right)$ is equal to the character of the quotient $\Omega_{i, N}$ of the space $\Omega_{i}$ by the ideal, generated by $a_{j}$ with $j>2 N$. Again, we have:

$$
\operatorname{ch} H\left(\mathcal{L}_{2 N}, V_{i}\right)=\operatorname{ch} \Omega_{i, N} \leq \operatorname{ch} \Omega_{i, N}^{\text {mon }}
$$

where $\Omega_{i, N}^{\text {mon }}$ is the quotient of $\Omega_{i}^{\text {mon }}$ by the ideal, generated by $a_{j}, j>$ $2 N$. Therefore,

$$
v_{N}^{i}=\operatorname{dim} H\left(\mathcal{L}_{2 N}, V_{i}\right) \leq \omega_{N}^{i}=\operatorname{dim} \Omega_{i, 2 N}^{\text {mon }} .
$$

We can easily calculate $\omega_{N}^{i}$ by induction [8].

Let $C_{i}^{r}=\left\{\left(m_{1}, \ldots, m_{l}\right) \mid m_{1} \geq \cdots \geq m_{l} \geq 1, m_{i} \geq m_{i+r-1}+\right.$ $\left.2, m_{l-i+1}>1\right\}$. The monomials

$$
\left\{a_{m_{1}} \ldots a_{m_{l}} \mid\left(m_{1}, \ldots, m_{l}\right) \in C_{i}^{r}\right\}
$$

constitute a linear basis in $\Omega_{i}^{\text {mon }}$.

For every integer $N>0$, introduce the subspaces $W_{k, N}^{i}, 1 \leq k \leq r$, of $\Omega_{i}^{\text {mon }}$, which are linearly spanned by the monomials

$$
\left\{a_{m_{1}} \ldots a_{m_{l}} \mid\left(m_{1}, \ldots, m_{l}\right) \in C_{i}^{r}, m_{1} \leq N, m_{k} \leq N-1\right\} .
$$

Clearly, $W_{1, N}^{i}$ is isomorphic to $\Omega_{i, N-1}^{\text {mon }}$.

The dimension of the space $W_{k, N}^{i}$, which is spanned by our monomials, in which $a_{N}$ is allowed in the power less than $k$, is equal to the sum of dimensions of the spaces $W_{l, N-1}^{i}$ with $l=1, \ldots, r-k+1$, because we have the relations $a_{N-1}^{u} a_{N}^{v}=0$ for $u+v=r$. Let us introduce the $r$-vector $\mathbf{w}_{N}^{i}$, whose components are $w_{k, N}^{i}=\operatorname{dim} W_{k, N}^{i}, k=1, \ldots, r$. We obtain the formula [8]:

$$
\mathbf{w}_{N}^{i}=M_{r} \mathbf{w}_{N-1}^{i}
$$

where $M_{r}$ is given by (2), which shows that $\mathbf{w}_{2 N+1}^{i}=M_{r}^{2 N} \mathbf{w}_{1}^{i}$.

Remark 1. As shown in [8], there is a $q$-deformation of this formula, which gives an expression for the characters of $W_{k, N}^{i}$.

By definition, $w_{k, 1}^{i}=\min \{i, k\}$. One can check that $\mathbf{w}_{1}^{i}=M_{r}^{2} \mathbf{x}_{i}$, and so $\mathbf{w}_{2 N+1}^{i}=M_{r}^{2 N+2} \mathbf{x}_{i}$. Therefore, $\omega_{N}^{i}=w_{1,2 N+1}^{i}=\mathbf{x}_{1}^{t} M_{r}^{2 N+2} \mathbf{x}_{i}$, and this gives us an upper bound for $v_{N}^{i}$.

\section{Proposition 4.}

$$
v_{N}^{i} \leq \mathbf{x}_{1}^{t} M_{r}^{2 N+2} \mathbf{x}_{i}
$$

But since $M_{r}^{t}=M_{r}$, this upper bound coincides with the lower bound from Proposition 3. Therefore, we have the equality

$$
u_{N}^{i}=v_{N}^{i}=\omega_{N}^{i}=\mathbf{x}_{1}^{t} M_{r}^{2 N+2} \mathbf{x}_{i} .
$$

This completes the proof of Theorem 1 .

Note that we have also proved that $\Omega_{i}$ is isomorphic to $\Omega_{i}^{\prime}=\mathbb{C}\left[a_{j}\right]_{j>0} / I_{i}$. 
Remark 2. For general minimal models the graded space $\Omega$ of an irreducible representation is also isomorphic to the quotient of $\mathbb{C}\left[a_{j}\right]_{j>0}$ by a certain ideal $I$. This ideal contains the operators $S_{n}$, given by formula (3) with $r=(p-1)(q-1) / 2$. They correspond to the action of the symbols of generators of the annihilating ideal of the minimal model. In general, however, these elements do not generate the ideal $I$. There are other generators, corresponding to the action of the symbols of other elements of the annihilating ideal on $\Omega$. It is an interesting problem to find explicit formulas for them. This will hopefully lead to a nice combinatorial description of general irreducible representations.

\section{Application: Gordon identities}

By formula (4), $\operatorname{dim} H\left(\mathcal{L}_{2 N}, V_{i}\right)(n) \leq \operatorname{dim} \Omega_{i, 2 N}^{\text {mon }}(n)$ for any $n$ and $N$. In the previous Section we proved that $\operatorname{dim} H\left(\mathcal{L}_{2 N}, V_{i}\right)=\operatorname{dim} \Omega_{i, 2 N}^{\text {mon }}$, therefore $\operatorname{dim} H\left(\mathcal{L}_{2 N}, V_{i}\right)(n)=\operatorname{dim} \Omega_{i, 2 N}^{\text {mon }}(n)$ for any $n$ and $N$. But, clearly, $\operatorname{dim} H\left(\mathcal{L}_{2 N}, V_{i}\right)(n)=\operatorname{dim} V_{i}(n)$ and $\operatorname{dim} \Omega_{i, 2 N}^{\operatorname{mon}}(n)=\operatorname{dim} \Omega_{i}^{\operatorname{mon}}(n)$ for $N$ large enough. Hence $\operatorname{dim} V_{i}(n)=\operatorname{dim} \Omega_{i}^{\operatorname{mon}}(n)$ for any $n$, and $\operatorname{ch} V_{i}=\operatorname{ch} \Omega_{i}^{\text {mon }}$. This can be interpreted as follows.

Proposition 5. The monomials

$$
\left\{L_{-m_{1}} \ldots L_{-m_{l}} v_{i} \mid\left(m_{1}, \ldots, m_{l}\right) \in C_{i}^{r}\right\}
$$

constitute a linear basis in the irreducible representation $V_{i}$ of the $(2,2 r+$ 1) minimal model.

Thus, we obtain the following formula for the character of the module $V_{i}$ :

$$
\operatorname{ch} V_{i}=\sum_{n \geq 0}\left|C_{i}^{r}(n)\right| q^{n}
$$

where $C_{i}^{r}(n)$ is the subset of $C_{i}^{r}$, which consists of the elements $\left(m_{1}, \ldots, m_{l}\right) \in$ $C_{i}^{r}$, for which $\sum m_{j}=n$. The right hand side of the formula (5) is known to be equal to

$$
\sum_{n_{1}, \ldots, n_{r-1} \geq 0} \frac{q^{N_{1}^{2}+\ldots+N_{r-1}^{2}+N_{i}+\ldots+N_{r-1}}}{(q)_{n_{1}} \ldots(q)_{n_{r-1}}},
$$

where $N_{j}=n_{j}+\ldots+n_{r-1}$, and $(q)_{n}=\prod_{l=1}^{l=n}\left(1-q^{l}\right)$ (cf. [9]).

On the other hand, it is known [10, 11] that

$$
\operatorname{ch} V_{i}=\prod_{n>0, n \neq 0, \pm i \bmod (2 r+1)}\left(1-q^{n}\right)^{-1} \text {. }
$$

This formula follows from the Weyl-Kac type character formula for irreducible minimal representations of the Virasoro algebra. 
Thus, we have obtained a new proof of the Gordon identities:

$$
\prod_{n>0, n \neq 0, \pm i \bmod (2 r+1)}\left(1-q^{n}\right)^{-1}=\sum_{n_{1}, \ldots, n_{r-1} \geq 0} \frac{q^{N_{1}^{2}+\ldots+N_{r-1}^{2}+N_{i}+\ldots+N_{r-1}}}{(q)_{n_{1}} \ldots(q)_{n_{r-1}}} .
$$

For $r=2$ these are the Rogers-Ramanujan identities:

$$
\prod_{n>0, n \neq 0, \pm 1 \bmod 5}\left(1-q^{n}\right)^{-1}=\sum_{n \geq 0} \frac{q^{n(n+1)}}{(q)_{n}} \text { and } \prod_{n>0, n \neq 0, \pm 2 \bmod 5}\left(1-q^{n}\right)^{-1}=\sum_{n \geq 0} \frac{q^{n^{2}}}{(q)_{n}} .
$$

They correspond to two irreducible representations of the $(2,5)$ minimal model.

Acknowledgements The main part of this work was done while B.F. was visiting the Isaac Newton Institute of the University of Cambridge. He would like to the thank the Institute for hospitality. E.F. thanks A.Szenes for valuable discussions.

\section{REFERENCES}

1. A.Belavin, A.Polyakov, A.Zamolodchikov, Infinite conformal symmetry in twodimensional quantum field theory, Nucl. Phys. B241 (1984) 333-380.

2. B.L.Feigin, D.B.Fuchs, Cohomology of some nilpotent subalgebras of the Virasoro and Kac-Moody algebras, in Geometry and Physics, Essays in honor of I.M.Gelfand on the occasion of his 75th birthday, eds. S.Gindikin and I.M.Singer, 209-235. North-Holland, 1991.

3. A.Beilinson, B.Feigin, B.Mazur, Introduction to algebraic field theory on curves, to appear.

4. B.Feigin, T.Nakanishi, H.Ooguri, The annihilating ideals of minimal models, Int. J. Mod. Phys. Suppl. 1A (1992) 217-238.

5. I.Frenkel, Y.Zhu, Vertex operator algebras associated to representations of affine and Virasoro algebras, Duke Math. Journal 66 (1992) 123-168.

6. B.Feigin, E.Frenkel, Affine Kac-Moody algebras at the critical level and GelfandDikii algebras, Int. J. Mod. Phys. Suppl. 1A (1992) 197-215.

7. I.Frenkel, Y.-Z.Huang, J.Lepowsky On axiomatic approaches to vertex operator algebras and modules, Preprint, 1990.

8. E.Frenkel, A.Szenes, Dilogarithm identities, q-difference equations, and the Virasoro algebra, Preprint hep-th/9212094, to appear in Int. Math. Res. Notices, Duke Math. Journal.

9. G.E.Andrews, The Theory of Partitions, Addison-Wesley, Reading, 1976.

10. V.Kac, M.Wakimoto, Modular invariant representations of infinite-dimensional Lie algebras and superalgebras, Proc. Natl. Acad. Sci. USA, 85 (1988) 4956-4960

11. V.Kac, Modular invariance in mathematics and physics, Address at the Centennial of the American Mathematical Society, Preprint, 1988. 
Landau Institute for Theoretical Physics, Moscow 117334, Russia

Department of Mathematics, Harvard University, Cambridge, MA 02138, USA 